\title{
Modulating molecular chaperones improves sensory fiber recovery and mitochondrial function in diabetic peripheral neuropathy
}

\author{
Michael J. Urban ${ }^{\mathrm{a}, \mathrm{b}}$, Pan Pan ${ }^{\mathrm{a}}$, Kevin L. Farmer ${ }^{\mathrm{a}}$, Huiping Zhao ${ }^{\mathrm{c}}$, Brian S.J. Blagg ${ }^{\mathrm{c}}$, and Rick \\ T. Dobrowsky,b, \\ aDepartment of Pharmacology and Toxicology, The University of Kansas, Lawrence, KS, USA \\ bProgram in Neuroscience, The University of Kansas, Lawrence, KS, USA \\ 'Department of Medicinal Chemistry, The University of Kansas, Lawrence, KS, USA
}

\section{Abstract}

\begin{abstract}
Quantification of intra-epidermal nerve fibers (iENFs) is an important approach to stage diabetic peripheral neuropathy (DPN) and is a promising clinical endpoint for identifying beneficial therapeutics. Mechanistically, diabetes decreases neuronal mitochondrial function and enhancing mitochondrial respiratory capacity may aid neuronal recovery from glucotoxic insults. We have proposed that modulating the activity and expression of heat shock proteins (Hsp) may be of benefit in treating DPN. KU-32 is a C-terminal Hsp90 inhibitor that improved thermal hypoalgesia in diabetic C57B1/6 mice but it was not determined if this was associated with an increase in iENF density and mitochondrial function. After 16 weeks of diabetes, Swiss Webster mice showed decreased electrophysiological and psychosensory responses and a >30\% loss of iENFs. Treatment of the mice with ten weekly doses of $20 \mathrm{mg} / \mathrm{kg} \mathrm{KU}-32$ significantly reversed preexisting deficits in nerve conduction velocity and responses to mechanical and thermal stimuli. KU-32 therapy significantly reversed the pre-existing loss of iENFs despite the identification of a sub-group of drug-treated diabetic mice that showed improved thermal sensitivity but no increase in iENF density. To determine if the improved clinical indices correlated with enhanced mitochondrial activity, sensory neurons were isolated and mitochondrial bioenergetics assessed ex vivo using extracellular flux technology. Diabetes decreased maximal respiratory capacity in sensory neurons and this deficit was improved following KU-32 treatment. In conclusion, KU-32 improved physiological and morphologic markers of degenerative neuropathy and drug efficacy may be related to enhanced mitochondrial bioenergetics in sensory neurons.
\end{abstract}

\section{Keywords}

Intra-epidermal nerve fibers; Sensory neurons; Nerve conduction velocity; Mitochondria; Bioenergetics; Respiratory capacity

\footnotetext{
(C) 2012 Published by Elsevier Inc.

Corresponding author at: Department of Pharmacology and Toxicology, The University of Kansas, 5064 Malott Hall, 1251 Wescoe Hall Dr., Lawrence, KS 66045, USA. Fax: +1 785864 5219. dobrowsky@ku.edu (R.T. Dobrowsky).

M.J.U. performed the animal studies; P.P. performed the iENF analysis, K.L.F. performed the bioenergetic analyses, H.Z. and B.S.J.B. provided KU-32; R.T.D. designed the research and wrote the manuscript. All authors reviewed and contributed to editing the manuscript.
} 
Diabetic peripheral neuropathy (DPN) is a common neurodegenerative complication of diabetes that has proven difficult to manage pharmacologically due to its multifactorial etiology (Yorek, 2011). Despite the efficacy of a plethora of small molecule inhibitors that target a single etiologic contributor to DPN in rodent models, the biochemical and temporal complexity underlying the progression of DPN in humans has proved challenging for translating the success in animal models to its clinical management (Calcutt et al., 2009). Thus, considerable need exists to identify novel pharmacologic targets or therapeutic paradigms that, in combination with good glycemic control, will help patients overcome the difficulties of slowing or reversing the progression of DPN. Examples of such approaches lie in improving dyslipidemia (Vincent et al., 2009), using a dual specificity vasopeptidase inhibitor to block angiotensin converting enzyme and neutral endopeptidase to improve neural and vascular deficits in DPN (Yorek, 2008) and the recent demonstration that hydroxyflavones may target multiple mechanisms that contribute to DPN (Stavniichuk et al., 2011). An additional and relatively unexplored paradigm is that pharmacologically modulating the activity of molecular chaperones will promote a broadly cytoprotective response that improves psychosensory, electrophysiological, biochemical and morphological indices of DPN.

Heat shock proteins 90 and 70 (Hsp90 and Hsp70) are two molecular chaperones that are critical for the proper folding of nascent proteins. Many neurodegenerative diseases (for example, Alzheimer's and Parkinson's disease) can be considered as protein-conformational disorders since the accumulation of specific mis-folded or aggregated proteins is a primary contributor to their etiology (Muchowski and Wacker, 2005). Although the accumulation of any one specific misfolded or aggregated protein is not associated with the development of DPN, hyperglycemia can increase the oxidative modification of amino acids (Akude et al., 2009; Obrosova, 2009) leading to impairments in protein folding (Muchowski and Wacker, 2005), increased interaction with molecular chaperones (Pratt et al., 2010) and decreased mitochondrial function (Fernyhough et al., 2010). Moreover, Hsp70 and Hsp90 are also components of the cellular heat shock response (HSR). Numerous conditions that promote cell stress lead to the Hsp90-dependent induction of the HSR which, in part, promotes the transient up-regulation of Hsp70 to aid the refolding or clearance of aggregated and damaged proteins (Pratt et al., 2010). Hsp70 upregulation can also prevent neuronal apoptosis (Bienemann et al., 2008) and decrease oxidative stress in neurodegenerative disorders (Chaudhury et al., 2006). Importantly, pharmacologic inhibitors ofHsp90 can induce Hsp70 and have shown potential in treating neurodegenerative diseases (Luo et al., 2007) and DPN (Urban et al., 2010).

Hsp90 contains a C-terminus ATP binding domain that weakly binds the antibiotic novobiocin (Marcu et al., 2000). Through systematic modification of the coumarin ring pharmacophore of novobiocin, KU-32 (Fig. 1) was identified as a C-terminal Hsp90 inhibitor whose ability to promote a heat shock response requires an acetamide substitution on the coumarin ring (Matts et al., 2011). Consistent with this effect, KU-32 protected against glucose-induced death of unmyelinated embryonic sensory neurons and ameliorated neuregulin-induced demyelination of myelinated Schwann cell and sensory neuron cocultures in an Hsp70 dependent manner (Urban et al., 2010). Importantly, KU-32 is readily bioavailable and weekly treatment of diabetic mice with $20 \mathrm{mg} / \mathrm{kg}$ KU-32 reversed multiple clinical indices of DPN, including thermal hypoalgesia. Loss of thermal sensation in the feet involves dysfunction or loss of small intra-epidermal nerve fibers (iENF) that respond to thermal stimuli (Beiswenger et al., 2008a, 2008b). Loss of iENFs has been reported in both Type 1 and Type 2 diabetics (Boucek et al., 2005; Pittenger et al., 2005) as well as individuals with impaired glucose tolerance (Smith et al., 2001). Thus, interventions that can improve fiber recovery or preserve the function of remaining fibers may be particularly beneficial to help manage human DPN. However, diabetic rodents can show pharmacologic 
recovery of thermal sensation in the absence of appreciable increases in iENF density (Obrosova et al., 2010; Stavniichuk et al., 2011). In our previous study, it was unclear if the recovery of thermal sensitivity after KU-32 treatment was associated with an increase in iENF density since untreated diabetic mice did not exhibit a significant loss of iENFs (Urban et al., 2010). Additionally, though the efficacy of KU-32 required expression of Hsp70, little insight was gained into how KU-32 may affect neuronal physiology. It is well appreciated that increased oxidative stress and mitochondrial dysfunction contribute to the pathogenesis of DPN (Chowdhury et al., 2011; Obrosova, 2009). We have shown recently that prolonged diabetes downregulated numerous mitochondrial proteins in dorsal root ganglia and that decreases in the mitochondrial proteome correlated with a decrease in mitochondrial respiratory capacity (Akude et al., 2011; Chowdhury et al., 2011). Therefore, the current study addressed whether the improvement in DPN by KU-32 is associated with a recovery of iENF density and an enhanced bioenergetic profile of adult sensory neurons using Swiss Webster mice as a genetically outbred strain that develops severe DPN and shows rapid changes in iENF density in response to STZ-induced diabetes (Beiswenger et al., 2008a, 2008b; Kennedy and Zochodne, 2005).

\section{Methods}

\section{Materials}

Streptozotocin (STZ), carbonylcyanide-4-(trifluoromethoxy)-phenylhydrazone (FCCP), oligomycin and poly-DL-ornithine were obtained from Sigma-Aldrich (St. Louis, MO). KU-32, [N-(7-((2R,3R,4S,5R)-3,4-dihydroxy-5-methoxy-6,6-dimethyl-tetrahydro-2Hpyran-2-yloxy)-8-methyl-2-oxo-2H-chromen-3-yl)acetamide], was synthesized and structural purity (>95\%) verified as described (Huang and Blagg, 2007). Collagenase and laminin were obtained from Gibco/Invitrogen (Carlsbad, CA).

\section{Induction of diabetes and drug treatments}

Six-week old, outbred Swiss Webster mice were purchased from Harlan Laboratories (Indianapolis, IN) and diabetes induced at 8 weeks of age. After $6 \mathrm{~h}$ of food withdrawal, mice were injected with $100 \mathrm{mg} / \mathrm{kg} \mathrm{STZ}$ dissolved in $0.2 \mathrm{ml}$ of sterile $0.1 \mathrm{M}$ sodium citrate in phosphate buffered saline. The STZ injection was repeated the next day and control mice received two injections of the vehicle. One week after the last injection, mice with fasting blood glucose (FBG) $>290 \mathrm{mg} / \mathrm{dl}$ (One-Touch Ultra glucometer) were deemed diabetic. After 16 weeks, control and diabetic animals were given a once per week intra-peritoneal injection ( $0.2 \mathrm{ml}$ ) of $5 \mathrm{mM}$ Captisol (CyDex Pharmaceuticals, Lenexa, KS) or $20 \mathrm{mg} / \mathrm{kg}$ KU-32 in $5 \mathrm{mM}$ Captisol for 10 weeks. All animals were maintained on a $12 \mathrm{~h}$ light/dark cycle with ad libitum access to water and Purina diet 5001 rodent chow. FBG and HbAlc levels $\left(\mathrm{A} 1 \mathrm{C} \mathrm{Now}{ }^{+}\right)$were determined prior to euthanizing the animals.

All animal procedures were performed in accordance with protocols approved by the Institutional Animal Care and Use Committee (IACUC) and in compliance with standards and regulations for care and use of laboratory rodents set by the National Institutes of Health. To comply with veterinary oversight and IACUC approval, we also employed a body condition score (BCS) to assess an overall decline in body condition that was unlikely to spontaneously improve (Ullman-Cullere and Foltz, 1999). In a mouse, a BCS1 indicates advanced muscular wasting and extreme loss of subcutaneous fat deposits yielding prominent indentations between vertebrae and sharp protuberances of spinal processes, the ileum and sacrum. This is an extreme that we tried to avoid since death would be imminent. A mouse of $\mathrm{BCS}^{+}$status had less severe fat loss and with milder bony protuberances. If the mouse showed hunched posture, closed or sunken eyes and was lethargic, this was all taken 
into consideration in conjunction with a veterinary consult to euthanize the animal since it was unlikely to spontaneously improve.

\section{Measures of nerve conduction velocity (NCV), mechanical and thermal sensitivity}

Motor (MNCV) and sensory (SNCV) NCV measurements were performed on deeply anesthetized mice using a TECA ${ }^{\mathrm{TM}}$ Synergy N2-EMG Monitoring System as we have previously described in detail (McGuire et al., 2009). A Dynamic Plantar Aesthesiometer (Stoelting Inc., Wood Dale, IL) fitted with a stiff monofilament was used to assess mechanical sensitivity. Preliminary experiments indicated that applying the filament to the plantar surface at an upward force of $10 \mathrm{~g}$ was necessary to provide a sufficient dynamic range to detect mechanical hypoalgesia in the diabetic Swiss Webster mice. Thermal sensitivity was assessed by paw withdrawal latency to a ramping focal radiant heat using a Hargreaves Analgesiometer (Stoelting Inc., Wood Dale, IL) (Hargreaves et al., 1988). Responses from each animal were measured 3-4 times on alternate feet and averaged (McGuire et al., 2009).

\section{Intra-epidermal nerve fiber analysis}

The plantar surface of both hind paws was dissected and placed in Zamboni's fixative overnight. Tissues were rinsed in PBS, cryoprotected in $30 \%$ sucrose overnight at $4{ }^{\circ} \mathrm{C}$, embedded in OCT, frozen on dry ice, and stored at $-80^{\circ} \mathrm{C}$. Frozen tissues were sectioned at $30 \mu \mathrm{m}$, placed on Superfrost Plus microscope slides, coded to obscure treatment and stored at $-80{ }^{\circ} \mathrm{C}$. Immunohistochemistry was performed using an anti ubiquitin C-terminal hydrolase (PGP 9.5) antibody (AbD Serotec, Oxford, UK) and the Vectastain Elite ABCPeroxidase kit (Vector Laboratories, Burlingame, CA). Slides were incubated in blocking buffer containing normal goat serum for $30 \mathrm{~min}$, incubated in a 1:1000 dilution of anti-PGP 9.5 antibody for $3 \mathrm{~h}$ at room temperature, rinsed in PBS, and incubated with secondary antibody for $1 \mathrm{~h}$ at room temperature. Slides were rinsed in PBS, incubated with an avidinbiotin complex solution (ABC solution) for $1 \mathrm{~h}$ at room temperature, rinsed and incubated in NovaRED peroxidase substrate solution for 2-3 min. Sections were counterstained with hematoxylin and a cover slip applied. Twelve digital images per animal were captured at $40 \times$ magnification using a Zeiss light microscope with a color CCD camera. Single nerve fibers crossing the dermal/epidermal junction were counted (intra-epidermal branches were disregarded) from each image by two observers blinded to treatment (Lauria et al., 2005). The iENF density was calculated by the number of fibers divided by the length of the dermal/epidermal junction (fibers $/ \mathrm{mm}$ ). The effect of the treatments on sub-epidermal fibers was estimated by tracing individual PGP 9.5-positive fibers and summing the fiber area using Image J. The percent of the dermal area occupied by the fibers was determined by dividing by the total sub-epidermal area.

\section{Isolation of adult sensory neurons}

Adult sensory neurons were isolated as described with minor modifications (Delree et al., 1989). Mice were euthanized via $\mathrm{CO}_{2}$ overdose, the spinal column dissected and the spinal cord dislodged with a gentle stream of PBS delivered with a $10 \mathrm{ml}$ syringe. The L4-L6 dorsal root ganglia (DRG) from 3 mice per treatment were removed and pooled together in Ham's F10 medium containing 10\% fetal calf serum(FCS) (Atlas Biologicals, Fort Collins, $\mathrm{CO}$ ). After removing connective tissue and trimming the nerve stumps, ganglia were placed in $1 \mathrm{ml}$ serum-free F10 medium. The cleaned ganglia were dissociated by the addition of 1 $\mathrm{ml}$ of $1.25 \%$ collagenase for $45 \mathrm{~min}$ at $37^{\circ} \mathrm{C}$ followed by a second digestion with $1 \mathrm{ml}$ of $2 \%$ trypsin for $30 \mathrm{~min}$ at $37^{\circ} \mathrm{C}$. Cells were isolated by centrifugation at $1000 \times g$ for $5 \mathrm{~min}$ and the pellet further dissociated by triturating in F10 medium with a fire-polished glass pipette. The cell suspension was layered on a $10 \mathrm{ml}$ gradient of sterile iso-osmotic Percoll

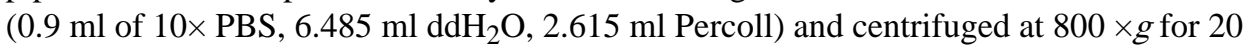


min to remove cell debris and myelin fragments. The cell pellet was resuspended in fresh F10 medium, passed through a $40 \mu \mathrm{m}$ nylon mesh and the filter washed with $5 \mathrm{ml}$ of serum free medium. The cells in the filtrate were recovered by centrifugation and resuspended in F10 medium (6.1 mM glucose) containing N2 supplement (Invitrogen) without insulin. Neurons were plated onto poly-DL-ornithine $(0.1 \mathrm{mg} / \mathrm{ml}$ overnight $) / \mathrm{laminin}(2 \mu \mathrm{g} / \mathrm{ml}$ for $3 \mathrm{~h})$ coated 96 well plates at $5 \times 10^{3}$ cells/well.

\section{Measuring mitochondrial respiration in intact adult sensory neurons}

Oxygen consumption rate (OCR) was assessed using intact adult DRG sensory neurons and an XF96 Extracellular Flux Analyzer (Seahorse Biosciences, North Billerica, MA) (Wu et al., 2007). Following isolation, adult sensory neurons were incubated for two days in F10 medium containing N2 supplement. Prior to assessing OCR, the neurons were placed in fresh bicarbonate-free DMEM containing $5.5 \mathrm{mMglucose}$ and incubated for $1 \mathrm{~h}$ at $37^{\circ} \mathrm{C}$. Baseline OCR was assessed in the XF96 analyzer using 4 measurement loops consisting of a 2 min mix cycle and a 5 min measurement cycle. Respiratory chain inhibitors were then sequentially injected into the wells and ATP-coupled oxygen consumption was calculated as the fraction of the basal OCR sensitive to $1 \mu \mathrm{g} / \mathrm{ml}$ oligomycin, an ATP synthase inhibitor. The maximal uncoupled respiration rate was determined by depolarizing the mitochondrial membrane potential with $1 \mu \mathrm{M} \mathrm{FCCP}$; inhibitor concentrations were optimized in preliminary experiments. After the respiratory measures, the cells were harvested and experimental rate values were normalized to protein content of each well. Maximal and spare respiratory capacities were determined from the rate data as described (Brand and Nicholls, 2011; Sansbury et al., 2011).

\section{Statistical analyses}

Data are presented as mean \pm SEM. After verifying equality of variance, differences between treatments were determined using a one or two-way ANOVA and Tukey's test; if appropriate, a non-parametric analysis was used.

\section{Results and discussion}

After 26 weeks of untreated diabetes, body weight was significantly decreased relative to control mice and as expected, both FBG and HbA1c were significantly elevated (Table 1). After 16 weeks of diabetes, KU-32 was administered weekly for 10 weeks and did not significantly change body weight, FBG or HbA1c levels relative to untreated diabetic animals. These results are similar to those observed in diabetic C57Bl/6 mice (Urban et al., 2010) and indicate that the dose and drug schedule are insufficient to improve overall glycemic control. It is interesting to note that during the period of KU-32 therapy, 7 of 15 untreated diabetic mice abruptly died (4) or required early euthanizing (3) for humane reasons due to a poor body condition score. However, only 3 of 14 diabetic animals receiving KU-32 were euthanized early due to a poor body condition score.

\section{KU-32 improves electrophysiologic, psychosensory and morphologic indices of DPN}

After 16 weeks of diabetes, MNCV decreased from $58.6 \pm 1.0$ to $48.9 \pm 2.0 \mathrm{~m} / \mathrm{s}(\mathrm{p}<0.001, \mathrm{n}$ $=4$ ) in the diabetic mice; the effect of diabetes on SNCV was more modest, $40 \pm 0.1$ to 36.2 $\pm 1.1 \mathrm{~m} / \mathrm{s}(\mathrm{p}<0.05, \mathrm{n}=4)$. After 26 weeks of untreated diabetes, both MNCV and SNCV remained significantly decreased compared to control (Fig. 2A). Although MNCV decreased another $15 \%(41.3 \pm 4.1 \mathrm{~m} / \mathrm{s})$ compared to the 16 week time point, weekly treatment with 20 $\mathrm{mg} / \mathrm{kg}$ KU-32 prevented this decline and reversed the pre-existing deficit in MNCV. Similarly, KU-32 also reversed the slowing observed in SNCV (Fig. 2A). 
Mechanical and thermal sensitivity were measured on alternate weeks beginning 4 weeks after the onset of hyperglycemia. After initiating KU-32 therapy, sensory assessments were then taken weekly. Although the force necessary to induce paw withdrawal remained around $5.5 \mathrm{~g}$ in control mice, diabetic Swiss Webster mice exhibited a significant decline in mechanical sensitivity after 4 weeks. This mechanical hypoalgesia steadily worsened until 18 weeks of diabetes and reached a plateau of around $9.5 \mathrm{~g}$ for the duration of the experiment (Fig. 2B). Relative to untreated diabetic mice, KU-32 induced a time-dependent improvement in mechanical sensitivity after 1 week of treatment. Following six weeks of drug treatment, the force required to induce paw withdrawal was indistinguishable from control animals. KU-32 had no effect on mechanical sensitivity in non-diabetic mice.

Diabetic Swiss Webster mice also showed a significant increase in paw withdrawal latency to a thermal stimulus after 5 weeks and this reached a plateau between 15 and 24 weeks (Fig. 2C). KU-32 treatment had no effect on thermal sensitivity in non-diabetic control mice but promoted a significant, time-dependent decrease in paw withdrawal latency in diabetic mice. However, this improvement was only indistinguishable from control animals after 10 weeks of treatment. Thus, although KU-32 therapy improved psychosensory detection of both mechanical and thermal stimuli, the slopes of the recovery curves support that the rate and magnitude of these changes differ.

Improvements in thermal sensitivity may occur in the absence of a recovery of iENFs (Beiswenger et al., 2008a, 2008b; Chen et al., 2005; Stavniichuk et al., 2011). Prior to drug treatment, diabetic Swiss Webster mice showed a 31\% decline in iENFs that crossed the dermal/epidermal junction $(n=4)$ (Figs. 3A and B). This deficit allowed us to assess whether modulating molecular chaperones may improve this morphologic index of DPN and assess its correlation with improved thermal sensitivity. At study termination, the decrease in iENF density in untreated diabetic mice was $29 \%$. Non-diabetic mice treated with KU-32 showed no difference in iENF density compared to control mice. In contrast, modulating molecular chaperones in the diabetic mice led to a partial, but significant, recovery of iENF density. Next, each animal's response latency to a thermal stimulus was plotted against its iENF density. Consistent with a prior study (Beiswenger et al., 2008a, 2008b), a significant inverse correlation was observed between untreated diabetic animals and both non-diabetic control groups ( $\mathrm{p}<0.0001, \mathrm{r}^{2}=0.6872$ ) (Fig. 3C). Compared to the untreated diabetic mice, all drug-treated diabetic animals showed a significant improvement in thermal sensitivity. However, k-means cluster analysis identified a subgroup of drug-treated diabetic animals that statistically clustered with the untreated diabetic mice. This subgroup of 4 mice showed significantly fewer fibers compared to the 7 remaining drug-treated diabetic mice $(\mathrm{p}<0.006$, Mann-Whitney test) but still showed a significant improvement in thermal hypoalgesia. Despite this group of non-responders, the iENF density in the remaining drug-treated diabetic animals was similar to the control groups. Unfortunately, the underlying reason why this subgroup did not respond to the drug is unclear. One possibility is that it may be related to the genetic heterogeneity associated with the outbred strain, but a larger sample size will be needed to determine if this may be the case. Nonetheless, these results strongly support that KU-32 therapy can reverse a pre-existing deficit in iENF density. Since the amount of fiber loss at 16 and 26 weeks in the untreated diabetic mice was similar, this would argue against the drug diminishing the rate of progressive fiber degeneration. Thus, KU-32 may improve thermal sensitivity by helping to promote small fiber regeneration and/or by decreasing the loss of TRPV1 heat-sensor receptors that is observed in diabetic mice with thermal hypoalgesia (Pabbidi et al., 2008). Unfortunately, the primary chaperone that mediates TRPV1 folding and trafficking is unknown (Niemeyer, 2005).

Lastly, we assessed whether the dermal nerve fibers were affected by the treatments. The percent area occupied by PGP9.5 positive dermal fibers was normalized to total dermal area 
and although diabetes trended toward decreasing dermal fiber area, no significant differences were observed (Veh + Veh, $5.9 \pm 1.0 \%$; Veh + KU-32, $5.7 \pm 1.9 \%$; STZ + Veh, $4.8 \pm 1.7 \%$; STZ + KU-32, $5.2 \pm 1.1 \%$ ).

\section{KU-32 improved mitochondrial bioenergetics in adult sensory neurons}

Enhanced oxidative stress and diminished mitochondrial function contribute to the pathogenesis of DPN (Chowdhury et al., 2011; Fernyhough et al., 2010; Obrosova, 2009). We have recently shown that numerous mitochondrial proteins are downregulated in dorsal root ganglia obtained from diabetic rats and that a decrease in mitochondrial respiration correlated with the diabetes-induced alterations in the mitochondrial proteome (Akude et al., 2011). Therefore, we examined whether the efficacy of KU-32 was associated with improving neuronal mitochondrial function.

Adult sensory neurons were isolated from lumbar DRG since they provide the axons for the myelinated and unmyelinated fibers that are affected in DPN, and whose physiology and morphology was improved by KU-32. An important advantage of adult sensory neurons is that they have been exposed to the physiological milieu of diabetes and exhibit molecular deficits associated with a "diabetic phenotype" (Huang et al., 2003, 2005; Vincent et al., 2010; Zherebitskaya et al., 2009). The ex vivo use of intact neurons also avoids artifacts associated with mitochondrial isolation from DRG. However, since the cultures are not pulsed with anti-mitotics, it is not possible to remove all contaminating Schwann cells and fibroblasts from the cultures (Fig. 4A), as is customary when using embryonic sensory neurons (Yu et al., 2008). Nevertheless, the enriched neuronal preparation readily elaborates neurites (Fig. 4A) and maintains a neuronal phenotype for a bioenergetic analysis as also reported by others (Chowdhury et al., in press).

Oxygen consumption rate (OCR) of intact adult sensory neurons was measured in real time using a XF96 Extracellular Flux Analyzer. An example of a typical mitochondrial function experiment of sensory neurons respiring in medium containing $5.5 \mathrm{mM}$ glucose is shown in Fig. 4B. The initial four rates provide a measure of the cells basal OCR (light blue shading); rate measures are expressed as a percent of the final basal OCR (rate 4) after normalizing to the total amount of protein per well. Respiratory chain poisons are used to assess mitochondrial dysfunction (Brand and Nicholls, 2011) and the addition of oligomycin (ATP synthase inhibitor) decreases the basal OCR. The portion of basal OCR that is coupled to ATP synthesis (dark blue shading) is estimated by the magnitude of this decrease and any residual OCR is from uncoupled respiration (proton leak). Maximal respiratory capacity (MRC, red shading) assesses the functional integrity of the respiratory chain once electron transfer is no longer limited by the proton gradient across the inner mitochondrial membrane (Brand and Nicholls, 2011). This is achieved following exposure to the protonophore, FCCP. Spare respiratory capacity (SRC) provides a functional indication of how close to its bioenergetic limit a cell is respiring and is estimated as the arithmetic difference between MRC and the basal OCR (purple shading) (Brand and Nicholls, 2011; Nicholls et al., 2007). A loss of SRC increases the susceptibility of cells to secondary stressors since it limits the dynamic range available to match energy output with environmental demands (Choi et al., 2009; Dranka et al., 2010). Thus, treatment-related differences in MRC and SRC reflect alterations in the bioenergetic limits and reserve capacity of the neurons. The green shading is representative of non-mitochondrial OCR values obtained after the addition of rotenone and antimycin A to inhibit complexes I and III, respectively.

At study termination, the neurons were isolated and used to assess OCR two days afterwards. After establishing the baseline OCR, the cells were treated with $1 \mu \mathrm{g} / \mathrm{ml}$ oligomycin to inhibit ATP synthase. In neurons obtained from animals treated with vehicle, the decrease in OCR indicated that $\sim 35 \%$ of basal respiration was directed toward ATP 
synthesis (Fig. 4C). In other words, this level of ATP-coupled respiration is sufficient to maintain ATP/ADP levels in control neurons. In contrast, the increase in oligomycinsensitive OCR in diabetic neurons suggests that more of the basal OCR is directed toward ATP synthesis. This may reflect the damaged electron transport capacity of the mitochondria which necessitate that more of the total OCR be directed toward ATP production to meet cellular demands. Importantly, neurons from diabetic mice treated with KU-32 showed a significant recovery in oligomycin-sensitive OCR with about $40 \%$ of the basal OCR now coupled to ATP production (Fig. 4C). Integrating the area under the curve for rates 4-7 indicated that diabetes significantly affected oligomycin-sensitive OCR relative to control and this was partially but significantly reversed in the KU-32 treated diabetic mice (Fig. 4D). Although FCCP stimulated MRC above baseline in neurons from both control and KU-32 treated animals, this response was blunted in diabetic neurons, consistent with the mitochondria being at their energetic limit. However, KU-32 treatment modestly but significantly, improved MRC as determined by integrating the area under the curve for rates 7-11 (Fig. 4D). However, in neither diabetic group did the MRC exceed the basal OCR (rate 3 ) as was seen in the control cells. Thus, the mitochondria have no SRC to aid the response to additional stress. That diabetes blunted the MRC and abolished SRC suggests that the cells are energetically stressed and that mitochondrial workload is increased. Although mitochondrial function was not completely recovered with KU-32 therapy, this nonetheless correlates with a reversal in the sensory deficits. Lastly, it should be noted that one limitation in our approach was the inability to assess non-mitochondrial OCR in the same well due to the limited number of cells and current technical limitations of the XF96 instrument. Although it is likely that non-mitochondrial OCR increased in the diabetic mice (Chowdhury et al., in press), this does not detract from the decrease observed in MRC and SRC.

\section{Conclusions}

The results from the current study support our proposal that modulating molecular chaperones may afford a novel approach to manage human DPN (Urban et al., 2010). The efficacy of the intervention strategy suggests that the ability of KU-32 to improve iENF density may be of clinical usefulness since abnormalities and loss of small sensory fibers can precede damage to larger myelinated fibers in human DPN (Malik et al., 2011). A second novel finding relates to the effect of KU-32 on improving mitochondrial function. After 26 weeks of diabetes, sensory neurons showed a markedly diminished maximal respiratory capacity which is consistent with previous reports that diabetes decreased numerous mitochondrial proteins, lowered respiratory rates (Akude et al., 2011; Chowdhury et al., 2010) and diminished both maximal and spare respiratory capacity in diabetic adult sensory neurons (Chowdhury et al., in press). KU-32 treatment modestly, but significantly, improved this deficit in respiratory capacity. However, further work will be necessary to determine if the partial recovery of mitochondrial function is a contributor to or consequence of the substantially improved sensory measures.

Although little is known on the effect of diabetes on Hsp70 expression and function in peripheral nerve, Hsp27 and Hsp70 were increased in DRGs after four months of diabetes in the Bio-breeding/Worcester (BB/Wor) rats, a model of type 1 diabetes (Kamiya et al., 2005). On the other hand, a decrease in Hsp70 levels in DRG correlated with the development of neuropathy and a loss of myelinated and unmyelinated fibers after ten months of hyperglycemia in BB/Wor rats, (Kamiya et al., 2006). However, decreased expression of Hsp70 is not essential for the development of DPN since diabetic mice lacking the inducible isoforms of Hsp70 (Hsp70.1 and 70.3) readily developed an insensate neuropathy (Urban et al., 2010). Conceivably, increased expression of Hsp70 may represent an early

cytoprotective response that dwindles due to the excessive metabolic disturbances associated 
with poorly controlled diabetes. Interestingly, a recent analysis of over 500 Type 1 diabetics from the EURODIAB Study found that elevated anti-Hsp70 antibody levels are associated with a 50\% lower likelihood of micro/macrovascular diabetic complications, independent of inflammation and other risk factors (Gruden et al., 2009). Although it remains unclear if Hsp70 antibodies contribute to diabetic complications or serve primarily as a biomarker, these data suggest that secretion of Hsp70 may be cytoprotective and associated with decreased risk of diabetic complications. Consistent with this possibility, neuronal uptake of Hsp70 secreted from glia decreased cell death induced by trophic factor withdrawal (Guzhova et al., 2001). Since diabetic Hsp70 knockout mice were unresponsive to KU-32 therapy, its neuroprotective properties hinge on the presence of Hsp70 (Urban et al., 2010). Together, these data support that modulating the expression of Hsp70 can ameliorate aspects of hyperglycemic stress and improve diabetic complications such as neuropathy.

It is well regarded that maintaining axonal plasticity of small epidermal fibers requires a continuous and available ATP supply (Chowdhury et al., 2011) and that defects in mitochondrial proteins such as bcl-w(Courchesne et al., 2011) can promote a sensory neuropathy. Thus, Hsp70 induction and improved mitochondrial bioenergetics may aid small fiber recovery. Consistent with this outcome, over-expression of Hsp70 in rat heart improved mitochondrial respiration and decreased mitochondrial damage in an ischemia/ reperfusion injury (Bu et al., 2002). Similarly, in mouse skeletal muscle, transgenic overexpression of Hsp70 enhanced mitochondrial enzyme activity (Chung et al., 2008). Under conditions of glucose-deprivation, Hsp70 overexpression in astrocytes inhibited proton leak in the electron transport chain and accumulation of reactive oxygen species (Ouyang et al., 2006). Notably, a folding competent chaperone may not be required for protection since an ATPase-deficient Hsp70 mutant was just as protective as the wild type protein (Ouyang et al., 2006). Thus, Hsp70 may function as part of a protein complex to affect mitochondrial physiology and recent work has uncovered that cytosolic Hsp70 and Hsp90 are part of a multiplex transporter that mediates mitochondrial import of nuclear-encoded proteins via interaction with Tom70 (Young et al., 2003). Mechanistically, it will be important to determine if the role of Hsp70 in aiding protein import into mitochondria (Schmidt et al., 2010 ) is related to improved mitochondrial function in sensory neurons and/or Schwann cells and the recovery of sensation.

In summary, pharmacologically targeting molecular chaperones can be sufficiently cytoprotective to reverse pre-existing functional and morphological deficits associated with insensate DPN in diabetic mice. The finding that KU-32 promotes significant morphologic recovery of iENFs argues against drug efficacy arising due solely to acute neurochemical corrections that are sufficient to improve NCV in rodents (Calcutt, 2010; Calcutt et al., 2009). Thus, modulating molecular chaperones may provide a novel therapeutic approach that enables neurons and their supporting cells to better tolerate glycemic insults that contribute to insensate neuropathy.

\title{
Acknowledgments
}

This work was supported by grants from the Juvenile Diabetes Research Foundation and The National Institutes of Health [NS054847] to RTD and [CA120458 and CA109265] to BSJB.

\section{Abbreviations}

\author{
DPN diabetic peripheral neuropathy \\ FBG fasting blood glucose \\ FCCP carbonylcyanide-4-(trifluoromethoxy)-phenylhydrazone
}




$\begin{array}{ll}\text { Hsp } & \text { heat shock protein } \\ \text { iENF } & \text { intraepidermal nerve fiber } \\ \text { MNCV } & \text { motor nerve conduction velocity } \\ \text { MRC } & \text { maximal respiratory capacity } \\ \text { SNCV } & \text { sensory nerve conduction velocity } \\ \text { SRC } & \text { spare respiratory capacity } \\ \text { STZ } & \text { streptozotocin }\end{array}$

\section{References}

Akude E, Zherebitskaya E, Roy Chowdhury SK, Girling K, Fernyhough P. 4-Hydroxy-2-nonenal induces mitochondrial dysfunction and aberrant axonal out-growth in adult sensory neurons that mimics features of diabetic neuropathy. Neurotox. Res. 2009; 1:28-38. [PubMed: 19557324]

Akude E, Zherebitskaya E, Chowdhury SKR, Smith DR, Dobrowsky RT, Fernyhough P. Diminished superoxide generation is associated with respiratory chain dysfunction and changes in the mitochondrial proteome of sensory neurons from diabetic rats. Diabetes. 2011; 60:288-297. [PubMed: 20876714]

Beiswenger KK, Calcutt NA, Mizisin AP. Dissociation of thermal hypoalgesia and epidermal denervation in streptozotocin-diabetic mice. Neurosci. Lett. 2008a; 442:267-272. [PubMed: 18619518]

Beiswenger KK, Calcutt NA, Mizisin AP. Epidermal nerve fiber quantification in the assessment of diabetic neuropathy. Acta Histochem. 2008b; 110:351-362. [PubMed: 18384843]

Bienemann AS, Lee YB, Howarth J, Uney JB. Hsp70 suppresses apoptosis in sympathetic neurones by preventing the activation of c-jun. J. Neurochem. 2008; 104:271-278. [PubMed: 17971127]

Boucek P, Havrdova T, Voska L, Lodererova A, Saudek F, Lipar K, Janousek L, Adamec M, Sommer C. Severe depletion of intraepidermal nerve fibers in skin biopsies of pancreas transplant recipients. Transplant. Proc. 2005; 37:3574-3575. [PubMed: 16298666]

Brand MD, Nicholls DG. Assessing mitochondrial dysfunction in cells. Bio-chem. J. 2011; 435:297312 .

Bu B, Klunemann H, Suzuki K, Li J, Bird T, Jin LW, Vincent I. Niemann-Pick disease type C yields possible clue for why cerebellar neurons do not form neurofibrillary tangles. Neurobiol. Dis. 2002; 11:285-297. [PubMed: 12505421]

Calcutt NA. Tolerating diabetes - an alternative therapeutic approach for diabetic neuropathy. ASN Neuro. 2010; 2:215-217.

Calcutt NA, Cooper ME, Kern TS, Schmidt AM. Therapies for hyperglycaemia-induced diabetic complications: from animal models to clinical trials. Nat. Rev. Drug Discov. 2009; 8:417-430. [PubMed: 19404313]

Chaudhury S, Welch TR, Blagg BS. Hsp90 as a target for drug development. Chem. Med. Chem. 2006; 1:1331-1340. [PubMed: 17066389]

Chen YS, Chung SSM, Chung SK. Noninvasive monitoring of diabetes-induced cutaneous nerve fiber loss and hypoalgesia in Thy1-YFP transgenic mice. Diabetes. 2005; 54:3112-3118. [PubMed: 16249433]

Choi SW, Gerencser AA, Nicholls DG. Bioenergetic analysis of isolated cerebrocortical nerve terminals on a microgram scale: spare respiratory capacity and stochastic mitochondrial failure. J. Neurochem. 2009; 109:1179-1191. [PubMed: 19519782]

Chowdhury SK, Zherebitskaya E, Smith DR, Akude E, Chattopadhyay S, Jolivalt CG, Calcutt NA, Fernyhough P. Mitochondrial respiratory chain dysfunction in dorsal root ganglia of streptozotocin-induced diabetic rats and its correction by insulin treatment. Diabetes. 2010; 59:1082-1091. [PubMed: 20103706] 
Chowdhury SKR, Dobrowsky RT, Fernyhough P. Nutrient excess and altered mitochondrial proteome and function contribute to neurodegeneration in diabetes. Mitochondrion. 2011; 11:845-854. [PubMed: 21742060]

Chowdhury SKR, Smith DR, Saleh A, Schapansky J, Marquez A, Gomes S, Akude E, Morrow D, Calcutt NA, Fernyhough P. Impaired amp-activated protein kinase signaling in dorsal root ganglia neurons is linked to mitochondrial dysfunction and peripheral neuropathy in diabetes. Brain. in press.

Chung J, Nguyen AK, Henstridge DC, Holmes AG, Chan MH, Mesa JL, Lancaster GI, Southgate RJ, Bruce CR, Duffy SJ, Horvath I, Mestril R, Watt MJ, Hooper PL, Kingwell BA, Vigh L, Hevener A, Febbraio MA. Hsp72 protects against obesity-induced insulin resistance. Proc. Natl. Acad. Sci. 2008; 105:1739-1744. [PubMed: 18223156]

Courchesne SL, Karch C, Pazyra-Murphy MF, Segal RA. Sensory neuropathy attributable to loss of bcl-w. J. Neurosci. 2011; 31:1624-1634. [PubMed: 21289171]

Delree P, Leprince P, Schoenen J, Moonen G. Purification and culture of adult rat dorsal root ganglia neurons. J. Neurosci. Res. 1989; 23:198-206. [PubMed: 2754765]

Dranka BP, Hill BG, Darley-Usmar VM. Mitochondrial reserve capacity in endothelial cells: the impact of nitric oxide and reactive oxygen species. Free Radic. Biol. Med. 2010; 48:905-914. [PubMed: 20093177]

Fernyhough P, Roy Chowdhury SK, Schmidt RE. Mitochondrial stress and the pathogenesis of diabetic neuropathy. Expert Rev. Endocrinol. Metab. 2010; 5:39-49. [PubMed: 20729997]

Gruden G, Bruno G, Chaturvedi N, Burt D, Pinach S, Schalkwijk C, Stehouwer CD, Witte DR, Fuller JH, Cavallo-Perin P. Group, E.P.C.S. Anti-hsp60 and anti-hsp70 antibody levels and micro/ macrovascular complications in type 1 diabetes: the EURODIAB Study. J. Intern. Med. 2009; 266:527-536. [PubMed: 19563392]

Guzhova I, Kislyakova K, Moskaliova O, Fridlanskaya I, Tytell M, Cheetham M, Margulis B. In vitro studies show that hsp70 can be released by glia and that exogenous hsp70 can enhance neuronal stress tolerance. Brain Res. 2001; 914:66-73. [PubMed: 11578598]

Hargreaves K, Dubner R, Brown F, Flores C, Joris J. A new and sensitive method for measuring thermal nociception in cutaneous hyperalgesia. Pain. 1988; 32:77-88. [PubMed: 3340425]

Huang Y-T, Blagg BSJ. A library of noviosylated coumarin analogues. J. Org. Chem. 2007; 72:36093613. [PubMed: 17328573]

Huang TJ, Price SA, Chilton L, Calcutt NA, Tomlinson DR, Verkhratsky A, Fernyhough P. Insulin prevents depolarization of the mitochondrial inner membrane in sensory neurons of type 1 diabetic rats in the presence of sustained hyperglycemia. Diabetes. 2003; 52:2129-2136. [PubMed: 12882932]

Huang TJ, Verkhratsky A, Fernyhough P. Insulin enhances mitochondrial inner membrane potential and increases ATP levels through phosphoinositide 3-kinase in adult sensory neurons. Mol. Cell. Neurosci. 2005; 28:42-54. [PubMed: 15607940]

Kamiya H, Zhangm W, Sima AAF. Apoptotic stress is counterbalanced by survival elements preventing programmed cell death of dorsal root ganglions in sub-acute type 1 diabetic BB/Wor rats. Diabetes. 2005; 54:3288-3295. [PubMed: 16249457]

Kamiya H, Zhang W, Sima AA. Degeneration of the Golgi and neuronal loss in dorsal root ganglia in diabetic Biobreeding/Worcester rats. Diabetologia. 2006; 49:2763-2774. [PubMed: 17047923]

Kennedy JM, Zochodne DW. Experimental diabetic neuropathy with spontaneous recovery: is there irreparable damage? Diabetes. 2005; 54:830-837. [PubMed: 15734862]

Lauria G, Cornblath DR, Johansson O, McArthur JC, Mellgren SI, Nolano M, Rosenberg N, Sommer C. EFNS guidelines on the use of skin biopsy in the diagnosis of peripheral neuropathy. Eur. J. Neurol. 2005; 12:747-758. [PubMed: 16190912]

Luo W, Dou F, Rodina A, Chip S, Kim J, Zhao Q, Moulick K, Aguirre J, Wu N, Greengard P, Chiosis G. Roles of heat-shock protein 90 in maintaining and facilitating the neurodegenerative phenotype in tauopathies. Proc. Natl. Acad. Sci. U.S.A. 2007; 104:9511-9516. [PubMed: 17517623]

Malik RA, Veves A, Tesfaye S, Smith G, Cameron N, Zochodne D, Lauria G. on behalf of The Toronto Consensus Panel on Diabetic, N. Small fibre neuropathy: role in the diagnosis of diabetic sensorimotor polyneuropathy. Diabetes Metab. Res. Rev. 2011; 27:678-684. 
Marcu MG, Chadli A, Bouhouche I, Catelli M, Neckers LM. The heat shock protein 90 antagonist novobiocin interacts with a previously unrecognized ATP-binding domain in the carboxyl terminus of the chaperone. J. Biol. Chem. 2000; 275:37181-37186. [PubMed: 10945979]

Matts RL, Brandt GE, Lu Y, Dixit A, Mollapour M, Wang S, Donnelly AC, Neckers L, Verkhivker G, Blagg BS. A systematic protocol for the characterization of Hsp90 modulators. Bioorg. Med. Chem. 2011; 19:684-692. [PubMed: 21129982]

McGuire JF, Rouen S, Siegfreid E, Wright DE, Dobrowsky RT. Caveolin-1 and altered neuregulin signaling contribute to the pathophysiological progression of diabetic peripheral neuropathy. Diabetes. 2009; 58:2677-2686. [PubMed: 19675140]

Muchowski PJ, Wacker JL. Modulation of neurodegeneration by molecular chaperones. Nat. Rev. Neurosci. 2005; 6:11-22. [PubMed: 15611723]

Nicholls DG, Johnson-Cadwell L, Vesce S, Jekabsons M, Yadava N. Bioenergetics of mitochondria in cultured neurons and their role in glutamate excitotoxicity. J. Neurosci. Res. 2007; 85:3206-3212. [PubMed: 17455297]

Niemeyer BA. Structure-function analysis of TRPV channels. Naunyn Schmiedebergs Arch. Pharmacol. 2005; 371:285-294. [PubMed: 15889240]

Obrosova IG. Diabetes and the peripheral nerve. Biochim. Biophys. Acta. 2009; 10:931-940. [PubMed: 19061951]

Obrosova IG, Stavniichuk R, Drel VR, Shevalye H, Vareniuk I, Nadler JL, Schmidt RE. Different roles of 12/15-lipoxygenase in diabetic large and small fiber peripheral and autonomic neuropathies. Am. J. Pathol. 2010; 177:1436-1447. [PubMed: 20724598]

Ouyang YB, Xu LJ, Sun YJ, Giffard RG. Overexpression of inducible heat shock protein 70 and its mutants in astrocytes is associated with maintenance of mitochondrial physiology during glucose deprivation stress. Cell Stress Chaperones. 2006; 11:180-186. [PubMed: 16817324]

Pabbidi RM, Yu SQ, Peng S, Khardori R, Pauza ME, Premkumar LS. Influence of TRPV1 on diabetes-induced alterations in thermal pain sensitivity. Mol. Pain. 2008; 4:9. [PubMed: 18312687]

Pittenger GL, Mehrabyan A, Simmons K, Amandarice, Dublin C, Barlow P, Vinik AI. Small fiber neuropathy is associated with the metabolic syndrome. Metab. Syndr. Relat. Disord. 2005; 3:113121. [PubMed: 18370718]

Pratt WB, Morishima Y, Peng HM, Osawa Y. Proposal for a role of the Hsp90/Hsp70-based chaperone machinery in making triage decisions when proteins undergo oxidative and toxic damage. Exp. Biol. Med. 2010; 235:278-289.

Sansbury BE, Jones SP, Riggs DW, Darley-Usmar VM, Hill BG. Bioenergetic function in cardiovascular cells: the importance of the reserve capacity and its biological regulation. Chem. Biol. Interact. 2011; 191:288-295. [PubMed: 21147079]

Schmidt O, Pfanner N, Meisinger C. Mitochondrial protein import: from proteomics to functional mechanisms. Nat. Rev. Mol. Cell Biol. 2010; 11:655-667. [PubMed: 20729931]

Smith AG, Ramachandran P, Tripp S, Singleton JR. Epidermal nerve innervation in impaired glucose tolerance and diabetes-associated neuropathy. Neurology. 2001; 57:1701-1704. [PubMed: 11706115]

Stavniichuk R, Drel VR, Shevalye H, Maksimchyk Y, Kuchmerovska TM, Nadler JL, Obrosova IG. Baicalein alleviates diabetic peripheral neuropathy through inhibition of oxidative-nitrosative stress and p38 MAPK activation. Exp. Neurol. 2011; 230:106-113. [PubMed: 21515260]

Ullman-Cullere MH, Foltz CJ. Body condition scoring: a rapid and accurate method for assessing health status in mice. Lab. Anim. Sci. 1999; 49:319-323. [PubMed: 10403450]

Urban MJ, Li C, Yu C, Lu Y, Krise JM, McIntosh MP, Rajewski RA, Blagg BSJ, Dobrowsky RT. Inhibiting heat shock protein 90 reverses sensory hypoalgesia in diabetic mice. ASN Neuro. 2010; 2:e00040. [PubMed: 20711301]

Vincent AM, Hayes JM, McLean LL, Vivekanandan-Giri A, Pennathur S, Feldman EL. Dyslipidemiainduced neuropathy in mice: the role of oxLDL/Lox-1. Diabetes. 2009; 58:2376-2385. [PubMed: 19592619] 
Vincent AM, Edwards JL, McLean LL, Hong Y, Cerri F, Lopez I, Quattrini A, Feldman EL. Mitochondrial biogenesis and fission in axons in cell culture and animal models of diabetic neuropathy. Acta Neuropathol. 2010; 120:477-489. [PubMed: 20473509]

Wu M, Neilson A, Swift AL, Moran R, Tamagnine J, Parslow D, Armistead S, Lemire K, Orrell J, Teich J, Chomicz S, Ferrick DA. Multiparameter metabolic analysis reveals a close link between attenuated mitochondrial bioenergetic function and enhanced glycolysis dependency in human tumor cells. Am. J. Physiol. Cell Physiol. 2007; 292:C125-C136. [PubMed: 16971499]

Yorek MA. The potential role of angiotensin converting enzyme and vasopeptidase inhibitors in the treatment of diabetic neuropathy. Curr. Drug Targets. 2008; 9:77-84. [PubMed: 18220715]

Yorek MA. Treatment of diabetic neuropathy with baicalein: intervention at multiple sites. Exp. Neurol. 2011; 232:105-109. [PubMed: 21907195]

Young JC, Hoogenraad NJ, Hartl FU. Molecular chaperones Hsp90 and Hsp70 deliver preproteins to the mitochondrial import receptor TOM70. Cell. 2003; 112:41-50. [PubMed: 12526792]

Yu C, Rouen S, Dobrowsky RT. Hyperglycemia and downregulation of caveolin-1 enhance neuregulin-induced demyelination. Glia. 2008; 56:877-887. [PubMed: 18338795]

Zherebitskaya E, Akude E, Smith DR, Fernyhough P. Development of selective axonopathy in adult sensory neurons isolated from diabetic rats: role of glucose-induced oxidative stress. Diabetes. 2009; 58:1356-1364. [PubMed: 19252136] 


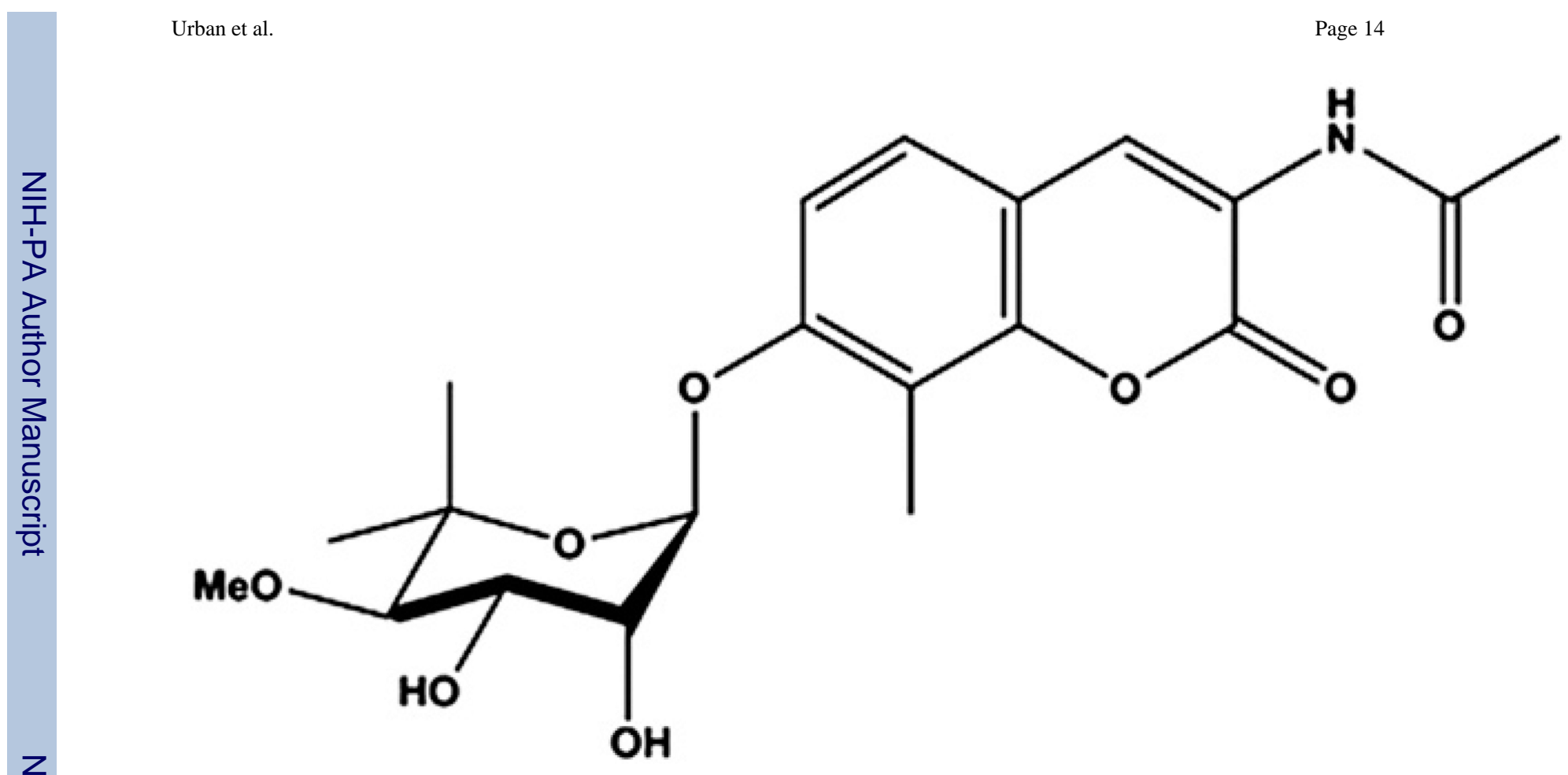

Fig. 1.

Structure of KU-32 

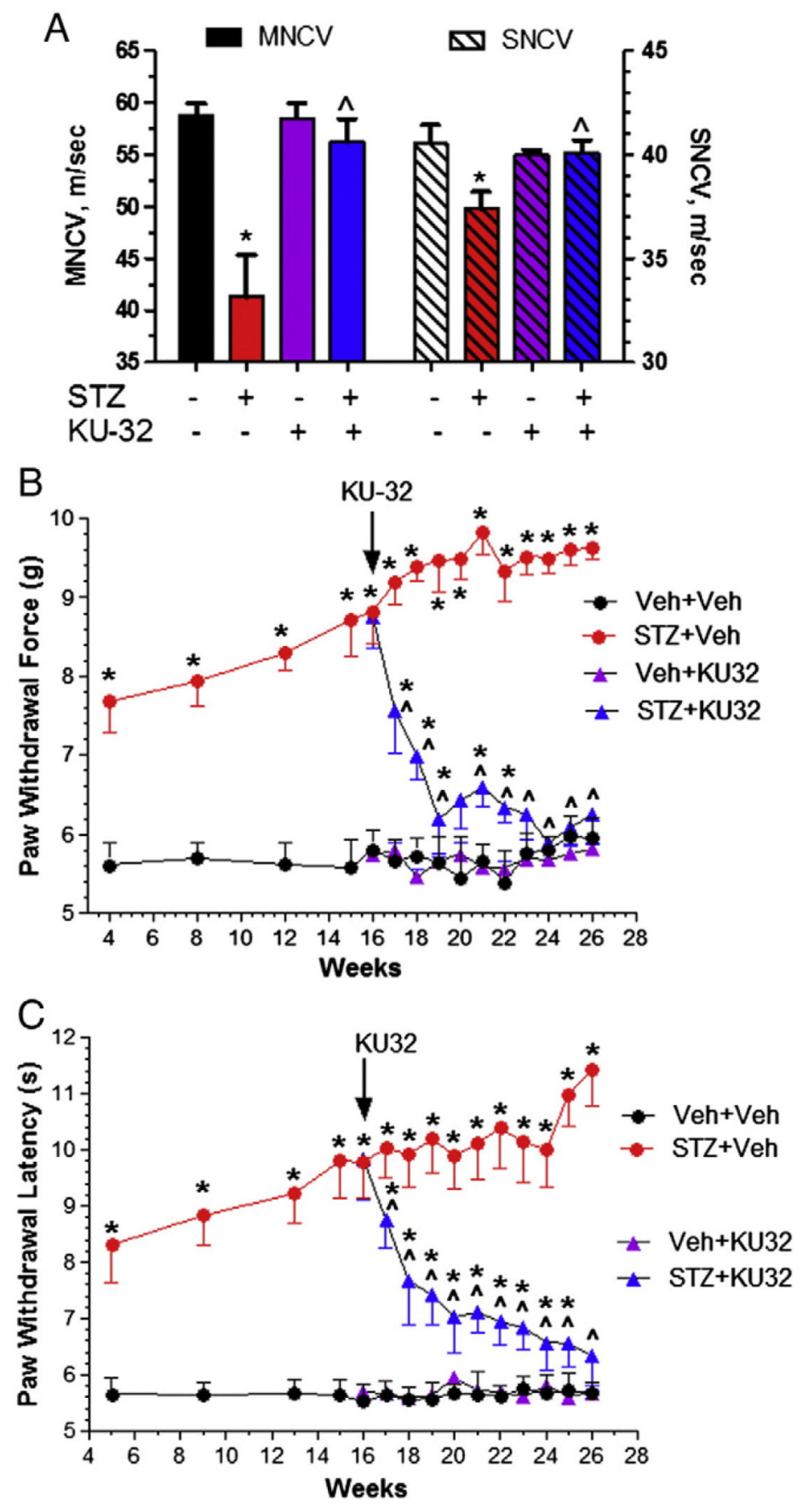

Fig. 2.

KU-32 reverses deficits in NCV and sensory hypoalgesia. Swiss Webster mice were rendered diabetic for 16 weeks and then treated with weekly doses of vehicle or $20 \mathrm{mg} / \mathrm{kg}$ KU-32 for 10 weeks. (A) Ten weeks of KU-32 treatment significantly improved diabetesinduced deficits in MNCV (solid bars, left axis) and SNCV (striped bars, right axis). *, $\mathrm{p}<0.05$ versus untreated control, ${ }^{\wedge}, \mathrm{p}<0.05$ versus $\mathrm{STZ}+\mathrm{Veh}$. Four weeks after the induction of diabetes, mechanical (B) and thermal (C) sensitivity were assessed biweekly. After initiating KU-32 therapy, sensory measures were taken weekly. Sixteen weeks of diabetes produced a significant decline in mechanical and thermal sensitivity which showed a time-dependent improvement following weekly treatment with $\mathrm{KU}-32$. * ${ }^{*} \mathrm{p}<0.05$ versus 
time-matched Veh $+\mathrm{Veh} ;{ }^{\wedge}, \mathrm{p}<0.05$ versus time matched STZ + Veh. Number of animals per treatment is given in Table 1 . 

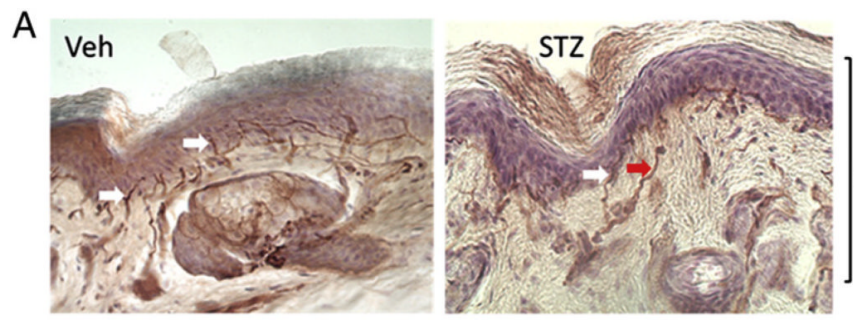

16 weeks
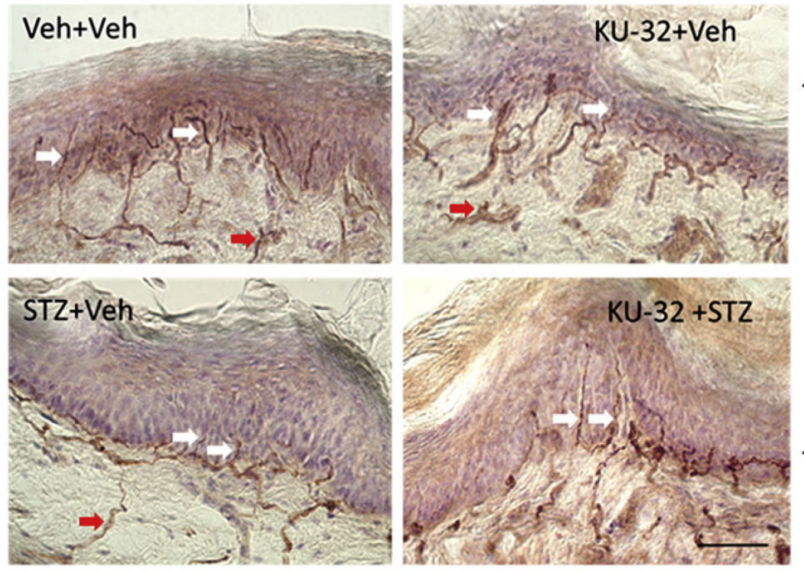

26 weeks

$\mathrm{B}$

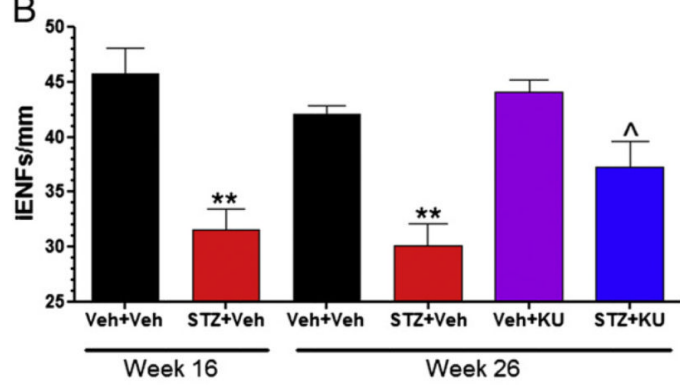

C

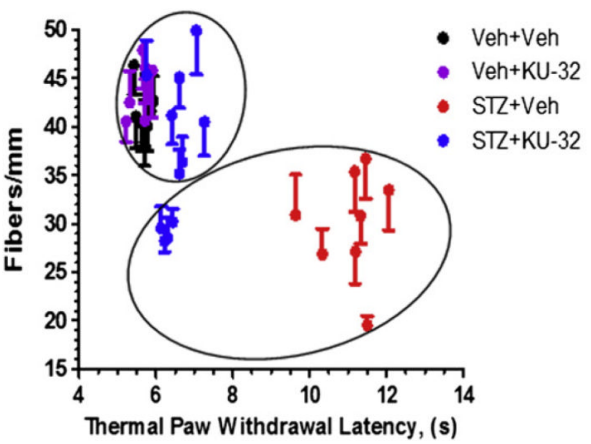

Fig. 3.

Diabetes-induced decrease in iENF density is improved by KU-32 treatment. Swiss Webster mice were rendered diabetic for 16 weeks, treated with weekly doses of vehicle or $20 \mathrm{mg} / \mathrm{kg}$ KU-32 for 10 weeks and iENF density determined. Representative images (A) and quantitative analysis (B) of iENF density. ${ }^{* *}, \mathrm{p}<0.01$ versus $\mathrm{Veh}+\mathrm{Veh} ;{ }^{\wedge}, \mathrm{p}<0.05$ versus $\mathrm{STZ}+$ Veh. Scale bar, $40 \mu \mathrm{m}$. Arrows show example of iENFs (white) and dermal nerve fibers (red) that were counted. (C) Thermal paw withdrawal latency was plotted against iENF density for each animal and subjected to k-means cluster analysis. Circles indicate cluster membership and identify that partial recovery of iENF density in diabetic mice 
treated with KU-32 was due to a subgroup in which recovery of thermal sensitivity and iENF density were dissociated. 


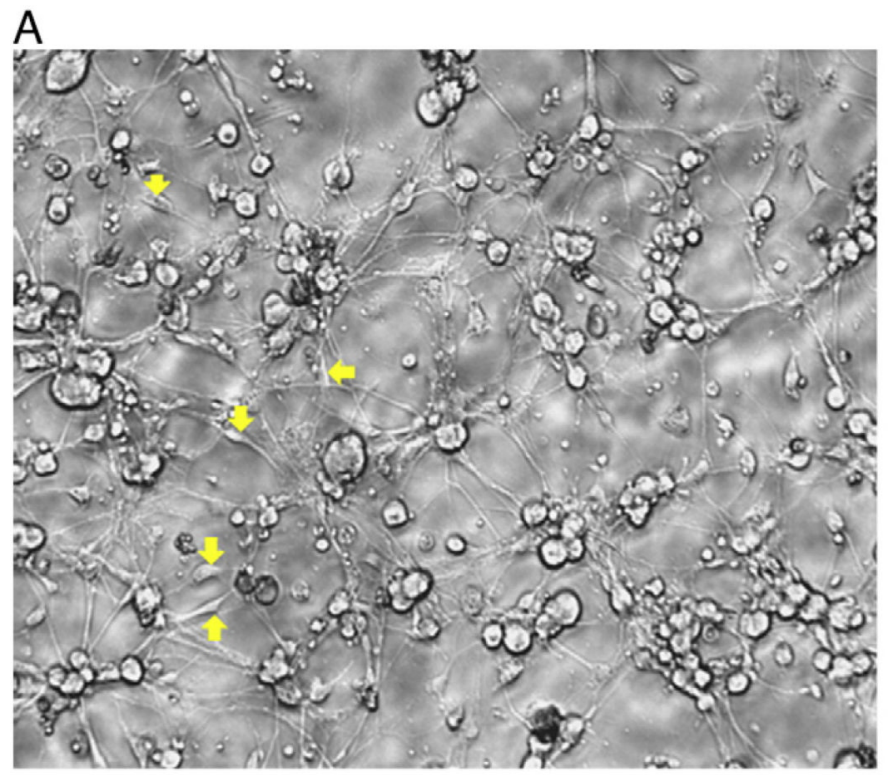

B
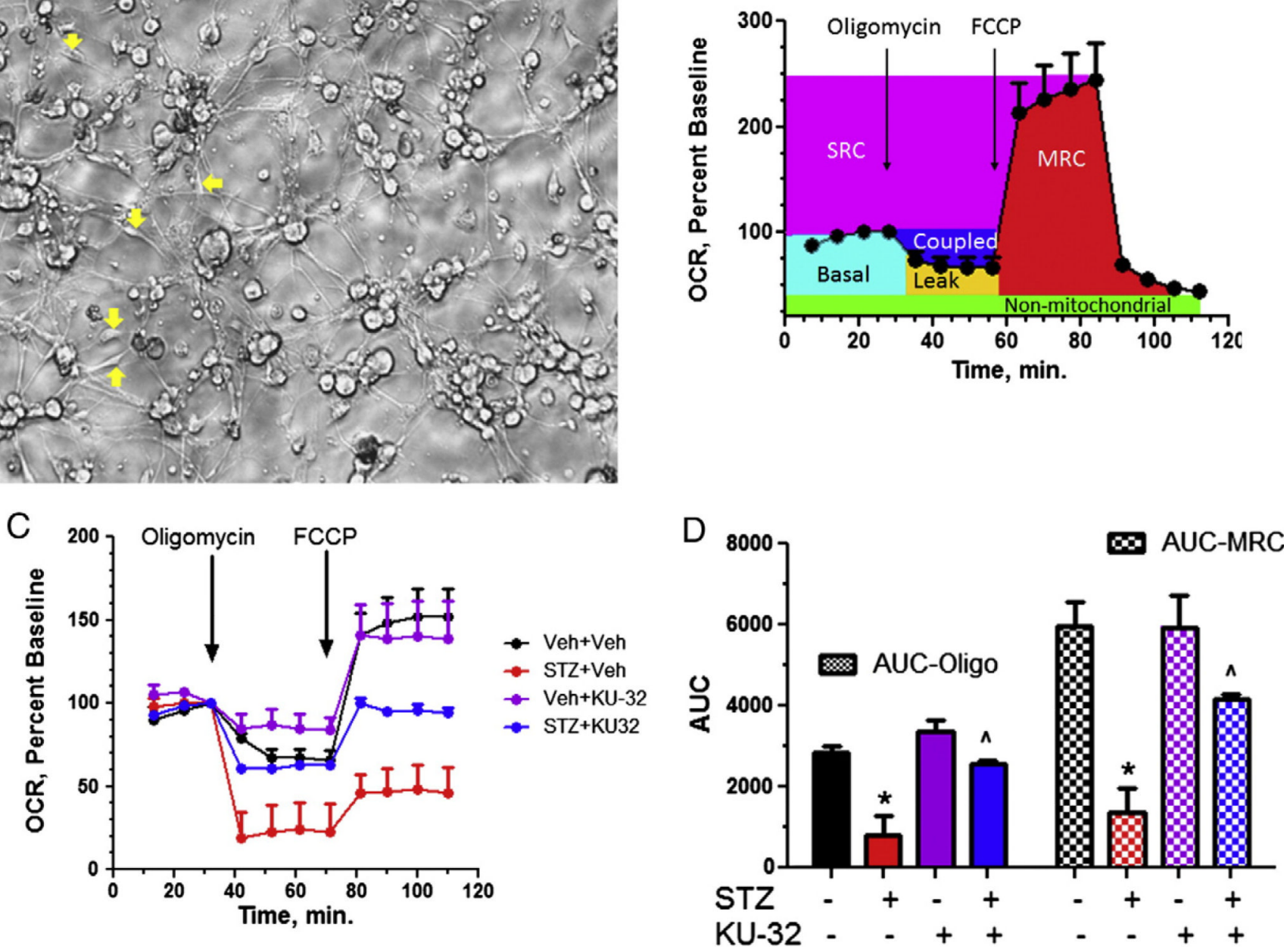

Fig. 4.

KU-32 improves mitochondrial bioenergetics in diabetic sensory neurons. Swiss Webster mice were rendered diabetic for 16 weeks, treated with weekly doses of vehicle or $20 \mathrm{mg} / \mathrm{kg}$ KU-32 for 10 weeks and sensory neurons purified from lumbar DRG. (A) Representative image of enriched culture of adult sensory neurons showing neurite outgrowth. Yellow arrows show examples of contaminating non-neurons. Scale bar, $40 \mu \mathrm{m}$. (B) Explanation of various measures of mitochondrial function in primary sensory neurons using the extracellular flux technology. Arrows show times of addition of oligomycin and FCCP. (C) Representative effect of oligomycin and FCCP on OCR in sensory neurons from obtained from the various treatment groups. (D) Diabetes significantly altered ATP-coupled respiration (solid bars) as determined by enhanced sensitivity to oligomycin and decreased MRC (hatched bars). Both these parameters were significantly improved in diabetic mice that received KU-32 therapy. *, p<0.05 versus time-matched Veh + Veh; ${ }^{\wedge}, \mathrm{p}<0.05$ versus time matched STZ + Veh. For interpretation of the references to color in this figure legend, the reader is referred to the web version of this article. 


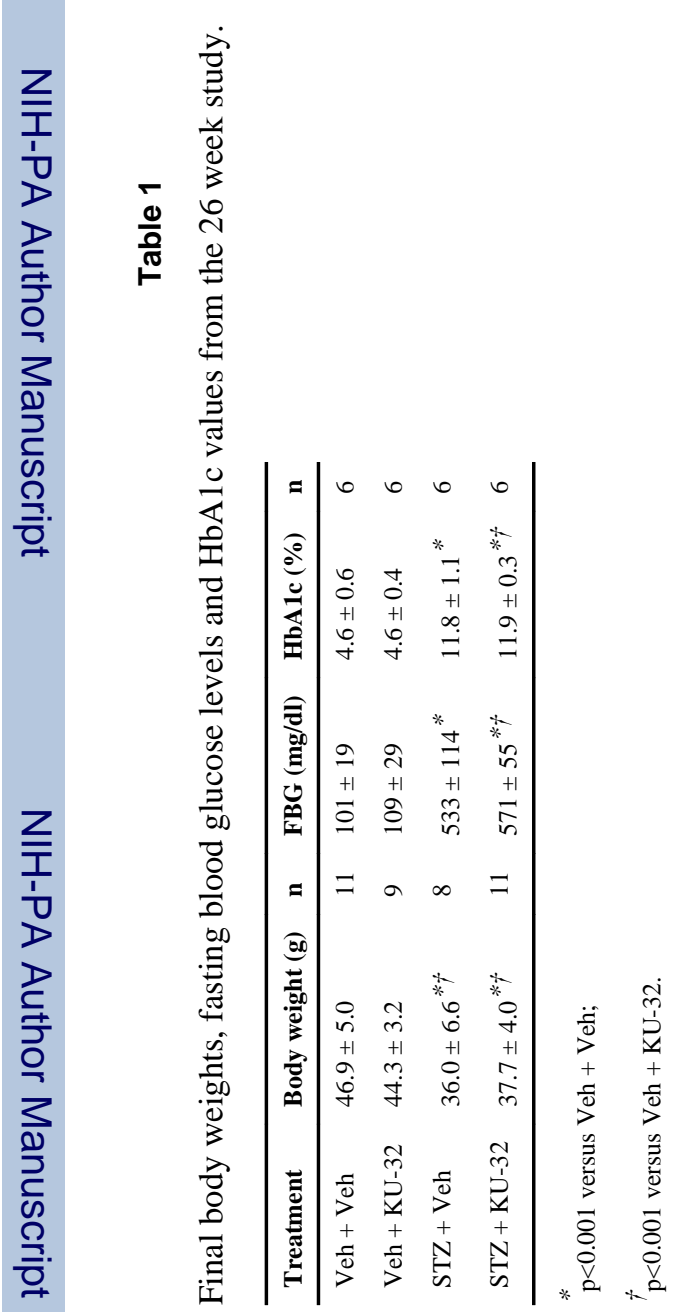

